\title{
Cavitation influence on hydroacoustic resonance in pipe
}

\author{
Nicolas Ruchonnet ${ }^{\mathrm{a}}$, Sébastien Alligné ${ }^{\mathrm{a}}$, Christophe Nicolet ${ }^{\mathrm{b}}$, François Avellan ${ }^{\mathrm{a}, *}$ \\ ${ }^{a}$ EPFL, Ecole polytechnique fédérale de Lausanne, Laboratory for Hydraulic Machines, avenue de Cour 33 bis, 1007 Lausanne, Switzerland \\ ${ }^{\mathrm{b}}$ Power Vision Engineering sàrl, Chemin des Champs-Courbes 1, 1024 Ecublens, Switzerland
}

\section{A R T I C L E I N F O}

\section{Article history:}

Received 7 November 2010

Accepted 6 October 2011

\section{Keywords:}

Hydroacoustics

Cavitation

Pressure fluctuations

Periodic vortex generation

Hydraulic machines and systems

\begin{abstract}
A B S T R A C T
In pipe systems, pressure and flow fluctuations below cutoff frequency propagate as plane waves along pipes. Depending on the pipe length and propagation velocity, resonance leading to high amplitude pressure fluctuation may occur. At low pressure, cavitation is an important source of fluctuation. Beside its active role in the mechanism of noise generation, the cavitation reflects partially the incoming plane waves. This may modify the values of the eigenfrequencies of the system consisting of the pipe, the contained fluid and the vapor cavity. The influence of cavitation is experimentally investigated in a hydroacoustic resonator: a straight pipe connecting two tanks. At three quarters of the pipe length, a bluff body is placed cross flow to generate periodic vortex wake cavitation in a limited section of the pipe. The analysis of the wall pressure measurements along the hydroacoustic resonator results is performed with the help of a one-dimensional transient model of the pipe including the compliance of the cavities created in the wake of the bluff body. The results of the numerical simulations enable the determination of both the eigenvalues within the resulting system of equations and the mode shape of the pressure fluctuations corresponding to the experimental results.
\end{abstract}

(c) 2011 Elsevier Ltd. All rights reserved.

\section{Introduction}

Cavitation plays an essential role in the generation and the propagation of acoustic waves in the hydraulic systems. It is of major concern for numerous industrial applications such as hydropower (Arpe et al., 2009), pumps (Berten et al., 2007), ship propellers (Watanabe and Brennen, 2003) and rocket propulsion (Bouziad, 2005; Bouziad et al., 2003). The generation and propagation of noise in pipes has been studied for a long period of time and is still topical (Eid and Ziada, 2011; Hachem and Schleiss, 2011). The review article of Reethof (1978) concerning noise induced by turbulence gives a good overview of the physical mechanism and the technical concerns. However, few studies involve the problematic nature of cavitation and mainly focus on noise generation, see Testud et al. (2007) and Hassis (1999); in their study of cavitation noise induced by single-hole and multi-hole orifices in pipes, Testud et al. (2007) observed eigenmodes in a domain bounded by the cavitating orifice and a valve containing air pockets. The air pockets and the vapor cavities were suspected to reflect the acoustic waves. As a result, the fluctuations were confined between those two reflective boundaries and eigenmodes have been observed in this restricted domain of the hydraulic circuit. In his experimental research, Hassis (1999) investigated the noise induced by a cavitating valve. His analysis included the effect of the vapor cavity on the pressure waves. He applied an analytical model to correlate the measured resonant frequencies upstream and downstream the valve with the help of the propagation velocity in the cavitating section of the pipe and its length.

\footnotetext{
* Corresponding author. Tel.: +412169325 24; fax: +41216933554.

E-mail address: francois.avellan@epfl.ch (F. Avellan).
} 


\begin{tabular}{|c|c|c|c|}
\hline & $K$ & vapor cavity compliance $\left(\mathrm{m} \mathrm{s}^{2}\right)$ \\
\hline \multicolumn{2}{|c|}{ Nomenclature } & \multicolumn{2}{|c|}{$A \quad$ pipe cross section $\left(\mathrm{m}^{2}\right)$} \\
\hline \multicolumn{2}{|c|}{$a_{0} \quad$ plane wave velocity $(\mathrm{m} / \mathrm{s})$} & $P_{y x}$ & cross power spectral density $\left(c_{p}^{2} / \mathrm{Hz}\right)$ \\
\hline & pipe width $(\mathrm{m})$ & $P_{x x}$ & power spectral density $\left(c_{\mathrm{p}}^{2} / \mathrm{Hz}\right)$ \\
\hline & flow velocity (m/s) & $p$ & pressure $(\mathrm{Pa})$ \\
\hline \multirow{2}{*}{$\begin{array}{l}c_{p} \\
D\end{array}$} & pressure coefficient & $p_{v}$ & water vaporization pressure $(\mathrm{Pa})$ \\
\hline & bluff body diameter (m) & $\mathrm{Re}$ & Reynolds number \\
\hline \multirow{2}{*}{$\begin{array}{l}D_{h} \\
f\end{array}$} & hydraulic diameter $(\mathrm{m})$ & St & Strouhal number \\
\hline & frequency $(\mathrm{Hz})$ & $T$ & frequency response function \\
\hline \multirow{2}{*}{$\begin{array}{l}f_{\text {cutoff }} \\
f_{D}\end{array}$} & pipe cutoff frequency $(\mathrm{Hz})$ & $V_{c}$ & vapor cavity volume $\left(\mathrm{m}^{3}\right)$ \\
\hline & drag frequency $(\mathrm{Hz})$ & $\lambda$ & wave length (m) \\
\hline$f_{L}$ & lift frequency $(\mathrm{Hz})$ & $\rho_{0}$ & water density $\left(\mathrm{kg} / \mathrm{m}^{3}\right)$ \\
\hline & pipe natural frequency $(\mathrm{Hz})$ & $\sigma$ & cavitation index \\
\hline & source frequency $(\mathrm{Hz})$ & $\sigma_{i}$ & incipient cavitation index value \\
\hline
\end{tabular}

In the present paper, the influence of hydrodynamic cavitation on pipe hydroacoustic resonance is investigated experimentally and numerically. The analysis is restricted to pressure fluctuation below cutoff frequency, $f_{\text {cutoff }}=$ $19000 \mathrm{~Hz}$ in the present experiment; accordingly plane wave propagation is assumed. The flow in a straight PVC pipe connecting two tanks at constant pressure has been investigated. At three quarters of the pipe length, a bluff body is placed cross flow to induce flow separation. The resulting flow instability, known as bluff body induced vortex shedding, generates acoustic pressure waves. For a sufficiently compact body, $D \ll \lambda$, the flow instability is equivalent to a dipole source of sound. In the framework of acoustic plane waves propagation in a pipe, this type of source is also known as momentum source and its amplitude and frequency are related to the drag force applied on the bluff body (Blake, 1986). Depending on the flow velocity, the frequency of the source may match one of the eigenfrequencies of the system and eventually lead to hydroacoustic resonance. The system refers to the hydroacoustic resonator including fluid compressibility and the effect of the pipe wall deformation. For a pure liquid, the propagation velocity of acoustic plane waves in a pipe is determined by the fluid compressibility and the wall stiffness (Ghidaoui et al., 2005; Nicolet, 2007; Tijsseling, 1996). Kortweg's equation (1) provides a good approximation of the propagation velocity of acoustic plane waves in an elastic pipe:

$$
\frac{1}{a_{0}^{2}}=\rho_{0}\left(\frac{1}{K_{w}}+\frac{\Delta A}{A \Delta p}\right),
$$

where the first term on the right accounts for the fluid compressibility while the second term accounts for the elastic deformation of the pipe.

At low pressure, cavitation is observed in the wake of the bluff body. In such condition, the amplitude of the acoustic fluctuation is strongly amplified in the entire pipe as the cavitation is an efficient source of sound. The corresponding source, interpreted as a mass source, is due to the variation of the vapor cavity volume (Blake, 1986). A strong coupling exists between the acoustic pressure fluctuation and the mass source in the cavitating region. This coupling results in the modification of the eigenfrequencies of the system. The hydrodynamic cavitation may therefore have two effects on the hydroacoustic resonance of the pipe. First, the cavitation can be an additional source of noise. Second, the eigenfrequencies of the system are modified by the cavitation. In the present study, it is found that the first effect could be neglected and we focus on the study of the second effect which consists mainly of a strong decrease of the pressure wave propagation velocity in the cavitating region of the pipe. The cavitating section being short with respect to the pipe length, a onedimensional mathematical model of the time dependent pipe flow using a lumped cavitation compliance can be successfully applied to describe the influence of cavitation on the eigenmodes of the system, see for instance Brennen and Acosta (1976) and Watanabe and Brennen (2003), who have applied such model to the cases of cavitating impeller in circuit and propeller resonance in a water tunnel.

The cavity compliance, $K$, defined as Eq. (2) is the partial derivative of the vapor volume, $V_{c}$, with respect to the pressure in the cavitating region (Brennen and Acosta, 1976; Rubin, 1966) and therefore accounts for the relation between the pressure in the source region and the mass source due to the vapor cavity:

$$
K=-\rho_{0} \frac{\partial V_{c}}{\partial p} .
$$

The paper is organized as follows. In Section 2, the hydroacoustic resonator and its instrumentation are introduced and the presentation of the investigated flow conditions is given as well. Then, in Section 3 the main results are presented, which contain the mass and the momentum source frequency, the influence of the cavitation index on the eigenfrequency of the system and the evaluation of the resonance frequency. In Section 4, the mathematical model is developed and 
numerical simulations are performed to analyze the influence of the cavitation index on the eigenfrequencies and eigenmodes of the system. Finally, in the last section, the conclusions of the performed work mentioned above are drawn.

\section{Experimental set-up}

The hydroacoustic resonator is made of a square cross-section of PVC pipe measuring $1.050 \mathrm{~m}$ in length, $0.040 \times 0.040 \mathrm{~m}^{2}$ for inner dimensions and a $2 \times 10^{-3} \mathrm{~m}$ wall thickness which connects two tanks. The flow perturbation is induced by a semi-circular bluff body of $20 \times 10^{-3} \mathrm{~m}$ diameter, located at three quarters of the pipe length. The bluff body is mounted perpendicular to the flow and is embedded on both sides of the pipe in a purposely designed Plexiglas support. The rounded side faces upstream and the flat side downstream. The flow velocity in the pipe is controlled by a variable-speed pump, see Fig. 1, and the hydrostatic pressure is adjusted, using a vacuum pump connected to the downstream tank. The water used during the experiments is air saturated tap water, and the water temperature is kept constant at $293 \pm 3 \mathrm{~K}$. The reflection condition of plane waves at both ends of the pipe is ensured by the free surface of the partially filled tanks. The influence of the free surface level on the resonance frequency has been verified and no significant effect has been noticed.

Particular attention has been paid to achieve a uniform flow at the pipe inlet. This is achieved with a special section placed in the upstream tank which consists of a settling chamber and a convergent, see Fig. 2. After leaving the upstream tank, the flow goes through a settling chamber featuring a honeycomb of $5 \times 10^{-3} \mathrm{~m}$ hexagonal mesh and a screen with $10 \times 10^{-3} \mathrm{~m}$ holes for an open area ratio of $1 / 4$. Then it flows to a convergent of $1 / 25$ cross-section ratio before entering the squared pipe. The transition between the outlet of the pipe and the downstream tank consists of an abrupt change of cross-section area, the area ratio being 2.4 .

The flow velocity is monitored with a vortex flow meter of 0 to $20 \times 10^{-3} \mathrm{~m}^{3} / \mathrm{s}$ range placed between the circulating pump and the upstream tank. Visualization of the wake cavitation is made with a high speed camera of $640 \times 512$ pixels resolution, 3000 frames/s acquisition rate and $80 \mu$ s exposure time. For the high speed visualization, the bluff body support is made of Plexiglas elements of $40 \times 10^{-3} \mathrm{~m}$ wall thickness and $70 \times 10^{-3} \mathrm{~m}$ total length fitted to the squared PVC pipe. For measuring the mode shape of the pressure fluctuations, flush mounted piezoresistive pressure sensors are distributed along the hydro-acoustic resonator; positions are given in Fig. 2. The sensor located in the downstream tank is not indicated in the figure. The sensor located below the mid-span trailing edge of the bluff body is also used for measuring

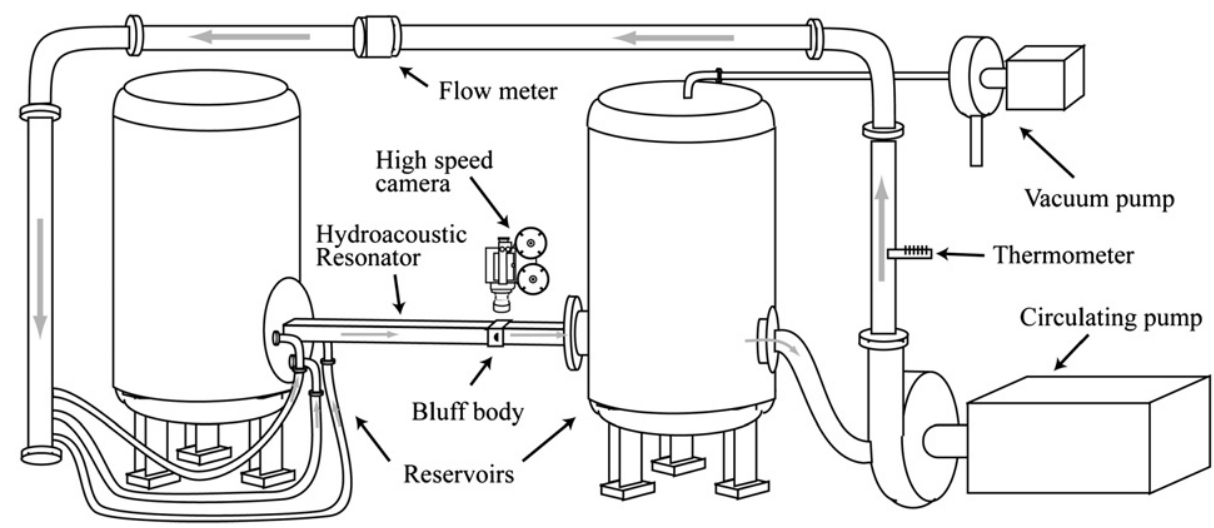

Fig. 1. Experimental set-up.

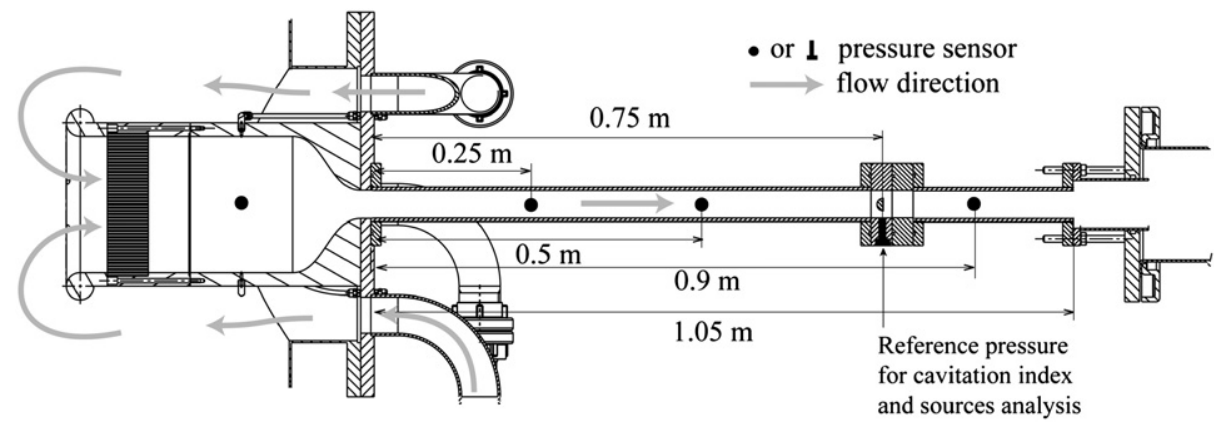

Fig. 2. Cross-sectional view of the hydroacoustic resonator with pressure sensors locations. 
the vortex shedding frequency. Static calibration of all sensors has been performed for absolute pressures ranging from 0.2 to $1.8 \times 10^{5} \mathrm{~Pa}$. The sensor dynamic response has been validated in previous experimentation by Farhat et al. (2002).

After conditioning and amplification, the signals of the 5 pressure sensors are simultaneously digitalized with a NI DAQ6221 at $1000 \mathrm{~Hz}$ sampling frequency. Welch's (1967) method is applied for estimating the power density spectrum, see Table 1.

The fluctuation frequency of the vapor volume is estimated using video analysis, the position of the camera being given in Fig. 1. To identify the gaseous phase, a motion detection algorithm has been used, see Fig. 3 . The method is based on the comparison of two successive images. Since the liquid phase is transparent and only the gaseous phase is visible, the cavity is identified as the moving part of the images. Then, the fluctuation frequency of the cavity volume is assumed to be the same as the fluctuations of the cavity area computed from the images.

Measurements have been performed at constant flow velocity and cavitation index. Two methods have been used to change the flow conditions, see Fig. 4:

(1) At constant flow velocity, open symbol, the hydrostatic pressure in the pipe has been changed by decrements of $2000 \mathrm{~Pa}$ from atmospheric pressure to minimum pressure reachable with the vacuum pump. The process has been repeated for $C=2.5 \mathrm{~m} / \mathrm{s}$ to $C=5 \mathrm{~m} / \mathrm{s}$ by steps of $0.5 \mathrm{~m} / \mathrm{s}$.

(2) At constant cavitation index, solid symbol, the velocity has been changed by steps of $0.1 \mathrm{~m} / \mathrm{s}$. At each step, the hydrostatic pressure has been adjusted to maintain the cavitation index.

The measurements performed at constant flow velocity, open symbol, have been used exclusively to evaluate the source frequency and the eigenfrequencies of the system. As the flow velocity is proportional to the source frequency

Table 1

Welch's method parameters.

\begin{tabular}{ll}
\hline Parameter & Value \\
\hline Sampling frequency & $1000 \mathrm{~Hz}$ \\
Time signal duration & $66 \mathrm{~s}$ \\
Spectral resolution & $0.98 \mathrm{~Hz}$ \\
Window size & $2^{10}$ samples \\
Windows overlap & $2^{8}$ samples \\
Window type & Hamming \\
\hline
\end{tabular}
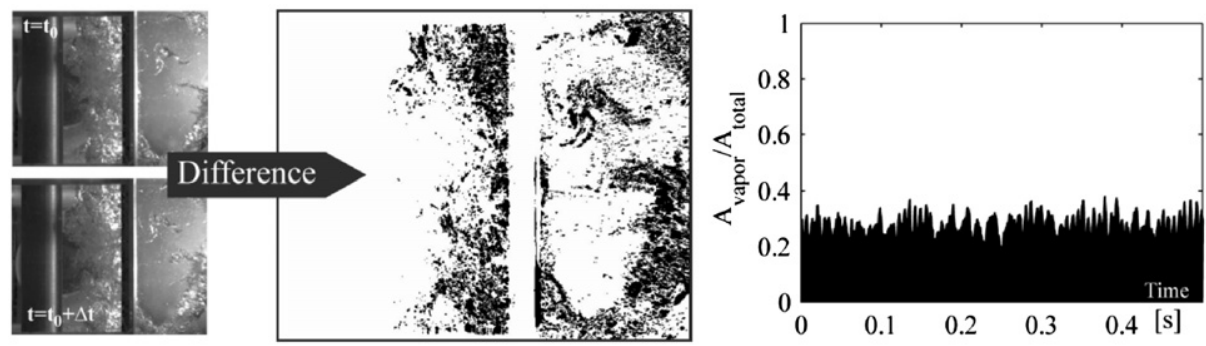

Fig. 3. Video analysis of the vapor fraction in the wake of the bluff body.

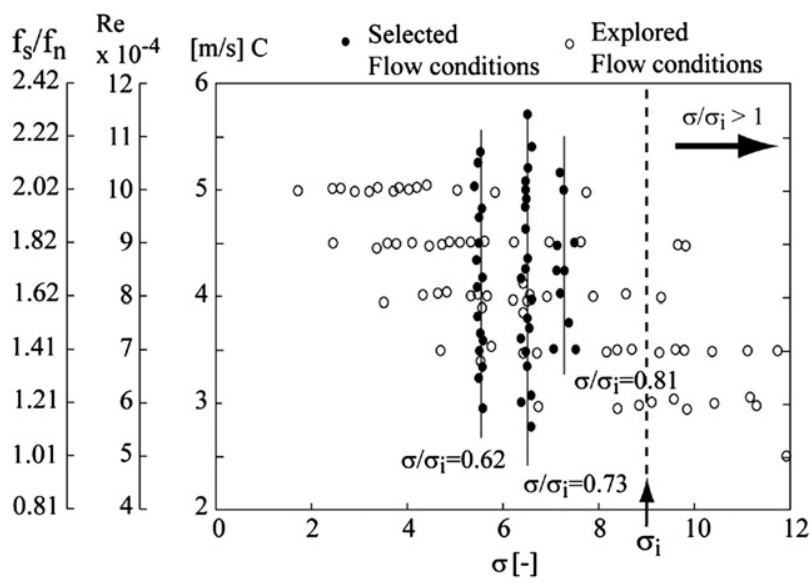

Fig. 4. Investigated flow conditions. 
according to the Strouhal number, the flow velocity is also presented in terms of source frequency in Fig. 4 . The measurements performed at constant cavitation index, solid symbol, also have been used to investigate the acoustic resonance; four cavitation indexes have been selected for this purpose. The flow conditions above incipient cavitation have been measured at atmospheric pressure and are therefore not visible in Fig. 4. For the three other flow conditions, vortex cavitation has been observed in the wake of the bluff body. The cavitation index $\sigma$ is defined as follows:

$$
\sigma=\frac{p-p_{v}}{(1 / 2) \rho C^{2}},
$$

where $p$ is the value of the static pressure under the bluff body. The pressure has been measured with the sensor located below the trailing edge of the bluff body, see Fig. $2 . p_{v}=2338 \mathrm{~Pa}$ is the water vaporization pressure at $293 \mathrm{~K}$ and $C$ is the pipe discharge velocity. The incipient cavitation index value $\sigma_{i}=9$, obtained from visualization has been used to scale the actual cavitation index; the selected values of scaled cavitation indexes being $>1,0.81,0.73$ and 0.62 .

Top views of the cavitation in the wake of the bluff body are presented in Fig. 5. The flow is from the left to the right. Large variations of cavity volume are observed for all $\sigma$. Nevertheless a clear increase of mean cavity volume is correlated with the decrease of $\sigma$. The spatial distribution of the cavitating vortices is weakly influenced by $\sigma$. From video analysis it can be stated that the vapor cavities are confined in a region located between $0.5 \mathrm{D}$ and $4 \mathrm{D}$ downstream the obstacle except for very low cavitation index $\left(\sigma / \sigma_{i}<0.5\right)$.

\section{Results}

\subsection{Sources of mass and axial momentum}

In hydroacoustics two types of sources are associated with specific hydrodynamic perturbations (Hirschberg and Rienstra, 2004). The momentum source is associated with perturbations induced by compact surfaces on flow at low Mach number, this type of source alters the conservation of momentum along the pipe axis (Curle, 1955; Inoue and Hatakeyama, 2002). The mass source is associated with the volume fluctuation of an external surface or a nonlinear density variation of the fluid, this type of source alters the mass conservation law (Williams, 1969).

In the present study, the momentum source is due to the drag force fluctuation on the bluff body as this component of the force is parallel to the direction of propagation of acoustic plane waves. The mass source is due to the volume fluctuation of the vapor cavity. The frequency of both sources is determined using the pressure sensor located under the bluff body. Indirect determination of the drag frequency is possible using the pressure field under the bluff body. In Fig. 6 , the time history of the pressure fluctuations under the bluff body is plotted using the pressure coefficient fluctuations $c_{p}^{\prime}$ defined as follows:

$$
c_{p}^{\prime}=\frac{p-\bar{p}}{(1 / 2) \rho C^{2}}
$$

with $\bar{p}$ the time averaged value of pressure.

In this region, the pressure time history is characterized by a periodic fluctuation with an unsteady low frequency modulation.

In Fig. 7(a), the frequency peaks of pressure fluctuation under the bluff body are plotted against the flow velocity for each investigated flow condition. Except from flow conditions corresponding to low cavitation index: $\sigma / \sigma_{i}>0.5$, a linear relation can be observed between the flow velocity and the frequency peak. Under the bluff body, the dominant pressure fluctuation frequency observed as a frequency peak is equal to the lift frequency.

The drag frequency is twice the lift frequency, according to Eq. (5) (Williamson, 1996):

$$
f_{S}=f_{D}=2 f_{L}=\frac{2 C}{D} S t
$$

where $D$ is the bluff body diameter, $C$ is the flow velocity and St the Strouhal number. A linear regression over the range $0.5<\sigma / \sigma_{i}<\infty$ yields the following estimations of the mean value and standard deviation, at $68 \%$ confidence interval, of the Strouhal number:

$$
\mathrm{St}=\frac{f_{L} D}{C}=0.390 \pm 0.006 \text {. }
$$

This particularly high value of St is due to the high confinement, $D / B=0.5$ (Ota et al., 1994). No variation of St is observed over the explored range of Reynolds number, Fig. 7(c). As shown in Fig. 7(b), the cavitation index also has no influence on the vortex shedding frequency for $\sigma / \sigma_{i}>0.5$. The Strouhal number increases solely for very low $\sigma$ values, as it has been obtained for a symmetric wedge (Young and Holl, 1966). The momentum source frequency is therefore linearly proportional to the flow velocity for the selected flow conditions.

The cavity pulsation frequency and the corresponding mass source frequency are measured via the following two methods:

- Video analysis by the image processing used for the vapor volume yielding the volume fluctuation frequency.

- Direct measure of the pressure fluctuation under the bluff body. 


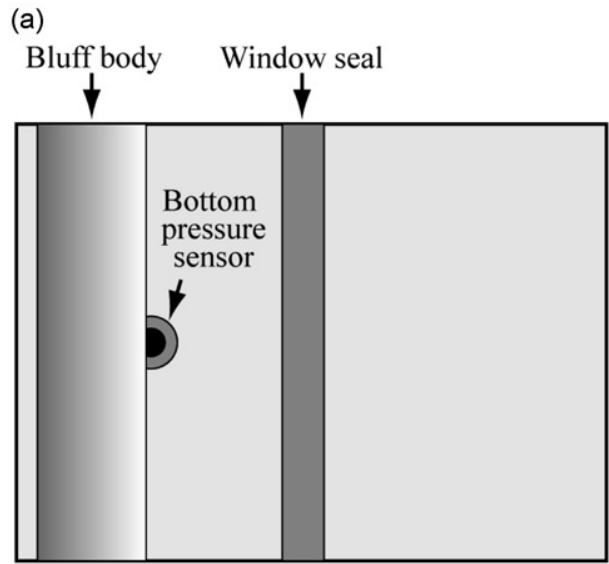

(b)

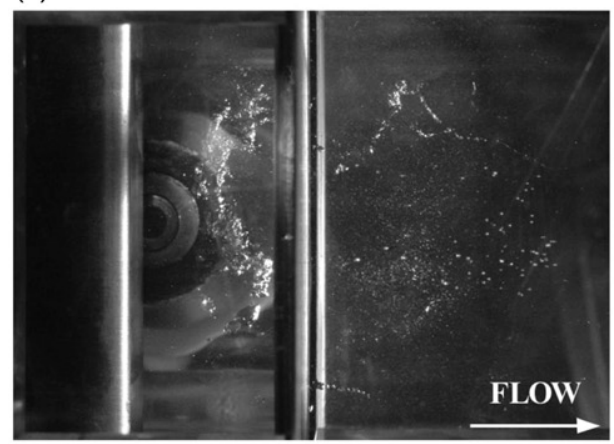

(c)

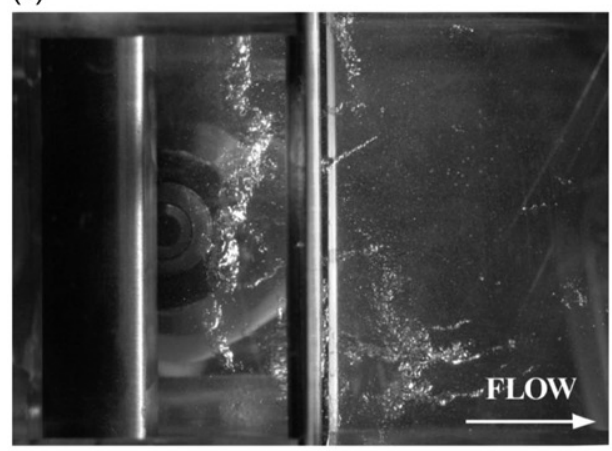

(d)

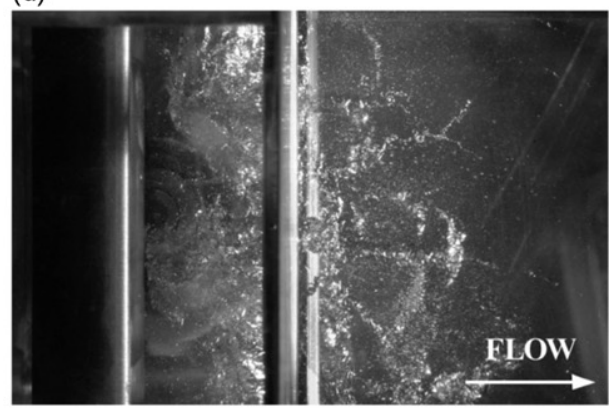

Fig. 5. Wake cavitation, (a) description of the image background, (b) $\sigma / \sigma_{i}=0.81$, (c) $\sigma / \sigma_{i}=0.73$ and (d) $\sigma / \sigma_{i}=0.62$.

In cavitating conditions, there are two peaks in the frequency spectrum of pressure fluctuation under the bluff body: a peak of large amplitude is observed at the lift frequency and a peak of smaller amplitude is observed at twice the lift frequency. The smaller one being not observed in cavitation free conditions, its occurrence is suspected to be due to the 


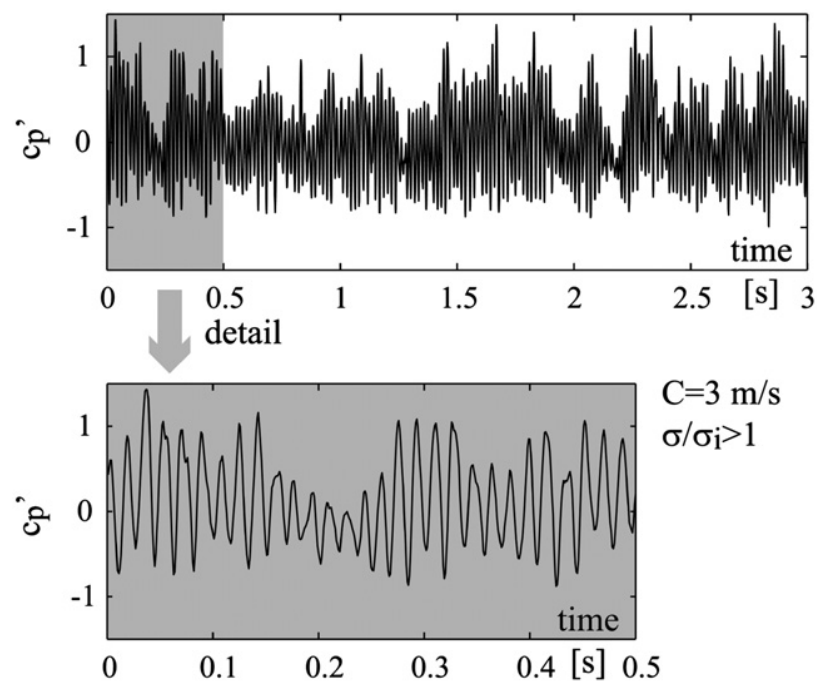

Fig. 6. Time history of pressure fluctuation under the bluff body for $C=3 \mathrm{~m} / \mathrm{s}$ and cavitation free flow condition.

acoustic fluctuations induced by the mass source. Since the pressure fluctuation due to the mass source is twice the lift frequency, by selecting the appropriate range of frequency in the spectrum of pressure fluctuation, it is possible to investigate the mass source exclusively.

In Fig. 8, an example of a waterfall diagram of Power Spectral Density (PSD) of pressure fluctuation is presented by varying the flow velocity and keeping constant the cavitation index at $\sigma / \sigma_{i}=0.62$. The dominant frequency, due to the lift, is not visible in this figure since $f_{L}<100 \mathrm{~Hz}$. Therefore, the frequency peak observed in the selected frequency range corresponds to the mass source frequency. The mass source frequency is proportional to the flow velocity. The large amplitude observed from $C=4 \mathrm{~m} / \mathrm{s}$ to $C=4.5 \mathrm{~m} / \mathrm{s}$ is due to the acoustic resonance.

In Fig. 9, both the mass source frequency evaluated using video analysis and pressure measurements are given. Pressure measurements corresponding to a specific range of cavitation index $0.5<\sigma / \sigma_{i}<0.72$ and to a quasi-constant flow velocity, open symbols in Fig. 4, are used to plot the error bar. The video analysis is performed for 5 flow conditions. Due to the short duration of the video record, $0.5 \mathrm{~s}$, the spectral resolution of the video analysis, $11.7 \mathrm{~Hz}$, is lower than the spectral resolution achieved with the pressure measurement, $0.98 \mathrm{~Hz}$. On one side, the direct determination using video analysis demonstrates the proportionality relation between the flow velocity and the mass source frequency, but the uncertainty of such method is large. On the other side, the indirect determination using pressure measurement is sufficiently accurate to confirm the relation between the frequency of the flow instability and the frequency of the mass source. However, the mass and momentum sources are both due to the flow instability in the wake of the bluff body. The frequency of this instability is proportional to the flow velocity and the ratio between the flow velocity and sources frequency is given by the Strouhal number see Eq. (4).

\subsection{System eigenmodes}

The broadband noise due to the low frequency modulation of the bluff body vortex shedding feeds acoustic energy on a large range of frequencies in the hydroacoustic resonator. This noise is exploited to detect the eigenfrequencies of the system.

In Fig. 10, a waterfall diagram of the PSD of the pressure fluctuation at $x / \mathrm{L}_{\mathrm{TOT}}=0.5$ is presented for various conditions of $\sigma$. The source frequency is selected in the range of $120 \mathrm{~Hz}\left(1.24 f_{n}\right)$ to $195 \mathrm{~Hz}\left(2.02 f_{n}\right)$, therefore the frequency peak due to the source is not visible. The eigenfrequency is identified as the frequency with maximum amplitude. Below $\sigma_{i}$, the first eigenfrequency decreases strongly with the cavitation index. Above $\sigma_{i}$, the eigenfrequency of the system is constant.

The average and standard deviation of the eigenfrequency of the system in cavitation-free conditions is given as follows:

$$
f_{n}=96.5 \mathrm{~Hz} \pm 1 \mathrm{~Hz}
$$

This value will be used as a reference frequency to normalize the source frequency and the resonance frequencies. Below $\sigma_{i}$, the eigenfrequency of the system is a nonlinear function of the cavitation index.

\subsection{Resonance}

The four waterfall diagrams of the PSD of pressure fluctuation at $x / L_{\mathrm{TOT}}=0.5$ are given in Fig. 11 by varying the flow velocity and keeping the value of the cavitation index constant for the 4 investigated cavitation indexes. According to 


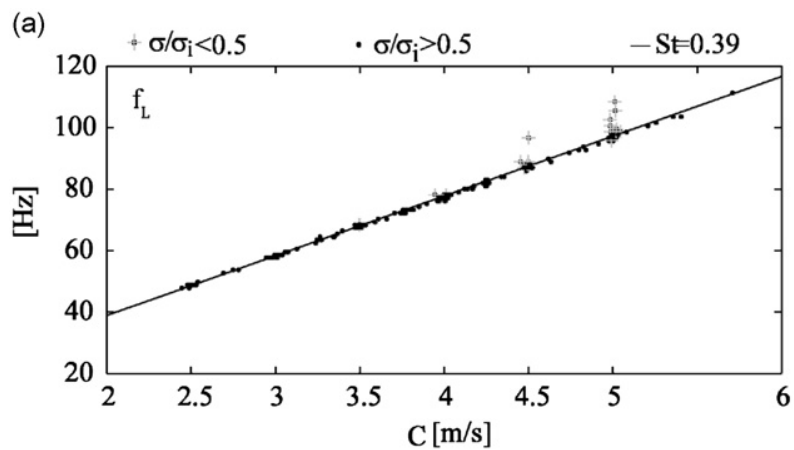

(b)

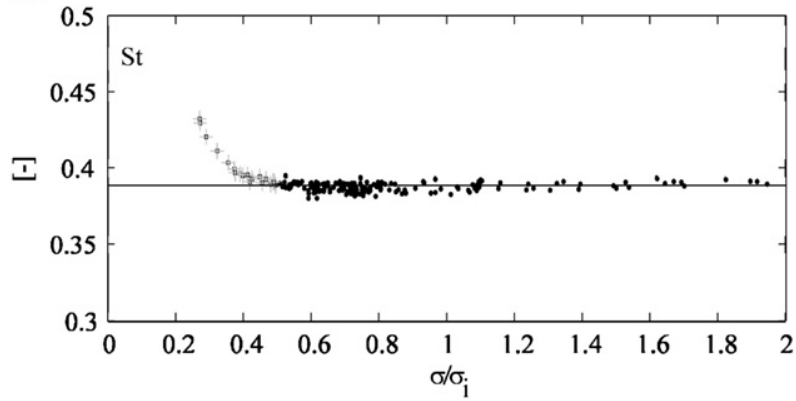

(c)

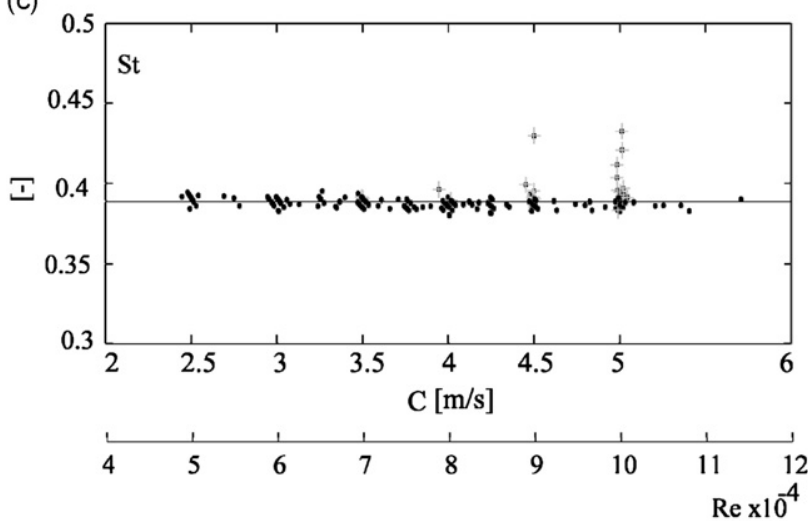

Fig. 7. (a) Lift frequency versus flow velocity, (b) Strouhal number versus cavitation index and (c) Strouhal number versus Re; points are measured values and line corresponds to $\mathrm{St}=0.39$.

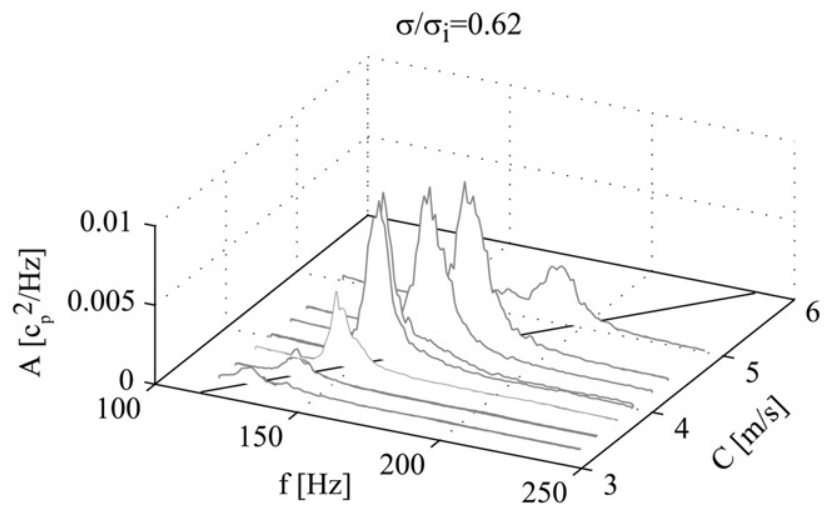

Fig. 8. Mass source frequency based on pressure fluctuation under the bluff body by keeping the cavitation index constant. 


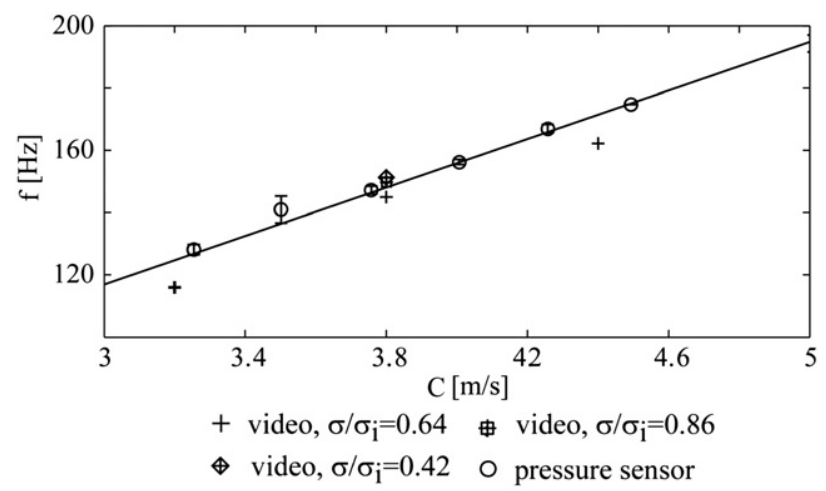

Fig. 9. Mass source frequency based on pressure fluctuation and video analysis.

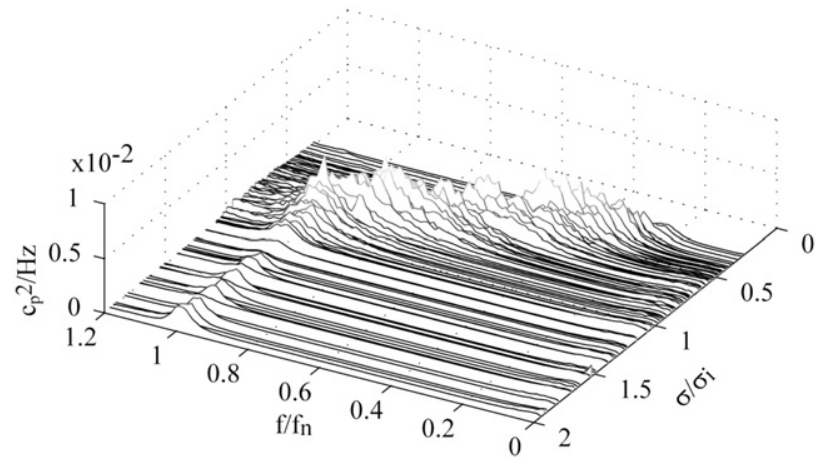

Fig. 10. Waterfall diagram of the PSD of pressure fluctuation at $x / L_{\mathrm{TOT}}=0.5$ by varying the cavitation index.

Eq. (5), the source frequency is derived from the flow velocity. The dashed line indicates the source frequency and the solid line, the eigenfrequency of the system. Maximum amplitude is reached at resonance as the source frequency is equal to one of the eigenfrequency values of the system. Similar behavior is observed for all sensors positions along the pipe. Nevertheless, the relative amplitudes along the pipe are determined by the eigenmodes, see Section 4 . Without cavitation, the 1st eigenfrequency of the system is evaluated at $96 \mathrm{~Hz}$, see Fig. 11(a). This value is in agreement with the evaluation based on the broadband noise excitation, see Section 3.2. For $\sigma / \sigma_{i}=0.81$, the resonant frequency is measured at $1.71 f_{n}$, see Fig. 11(b). As the onset and development of cavitation are expected to decrease the value of the eigenfrequency of the system, this resonant frequency may correspond to the second eigenfrequency. Lower resonant frequency values are measured for $\sigma / \sigma_{i}=0.73$ and $\sigma / \sigma_{i}=0.62$. For these values of cavitation index, the resonant frequencies decrease down to $1.56 f_{n}$ and $1.48 f_{n}$, respectively, see Fig. 11(c) and (d). It confirms the correspondence of the resonance with the 2nd eigenfrequency for cavitating flow conditions. At low flow velocity, the limited capacity of the vacuum pump prevents us to reach the cavitation conditions corresponding to resonance at the first eigenfrequency.

\section{Analysis}

\subsection{One-dimensional hydroacoustic model}

The model is a one-dimensional description of time dependent pipe flow (Ghidaoui et al., 2005; Nicolet, 2007):

$$
\frac{\partial p}{\partial t}+\rho_{0} a_{0}^{2} \frac{\partial C}{\partial x}=0, \quad \frac{\partial C}{\partial t}+\frac{1}{\rho_{0}} \frac{\partial p}{\partial x}+\frac{f\left|C_{0}\right|}{2 \sqrt{A_{0}}} C=0,
$$

where $p$ is the pressure, $C$ the discharge velocity, $C_{0}$ the reference discharge velocity, $\rho_{0}$ the water density, $a_{0}$ the propagation velocity, $A_{0}$ the cross section area, $f$ the friction factor, $t$ the time and $x$ the position along the pipe axis.

The pipe length corresponds to the experiment $\left(L_{\mathrm{TOT}}=1.05 \mathrm{~m}\right)$, no end correction is introduced. Constant reservoir pressure is imposed for both the inlet and outlet boundaries.

The measured values of the resonant frequency corresponding to cavitation-free conditions, without any vapor cavity, have been used to calculate the propagation velocity according to Wylie and Streeter (1993):

$$
2 L_{\mathrm{TOT}} f_{n}=a_{0}=203 \mathrm{~m} / \mathrm{s} \text {. }
$$


(a)

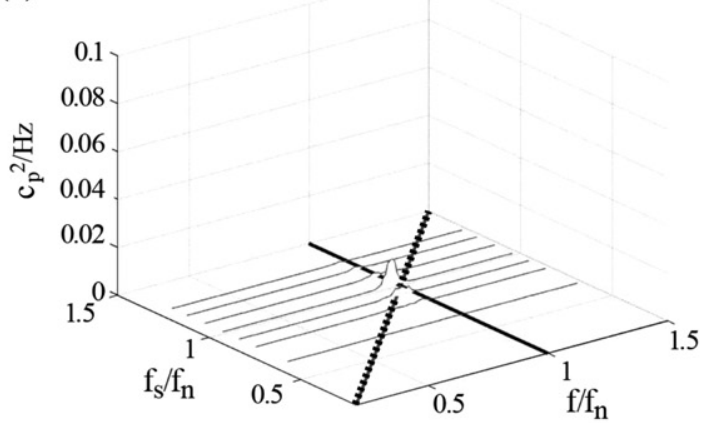

(b)

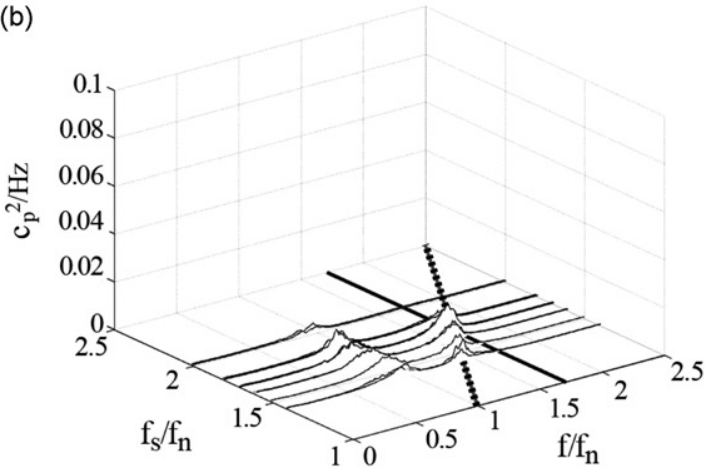

(c)

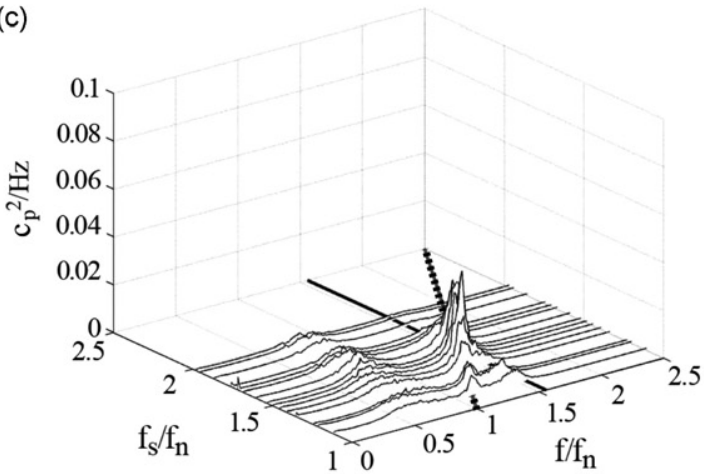

(d)

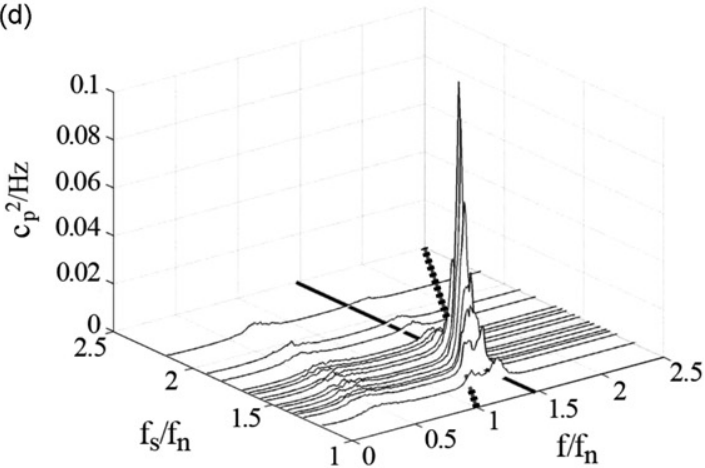

Fig. 11. PSD of $c_{p}$ at $x / L_{\mathrm{TOT}}=0.5$ versus flow velocity and equivalent source frequency for 4 cavitation indexes. (a) $\sigma / \sigma_{i}>1$; (b) $\sigma / \sigma_{i}=0.81$; (c) $\sigma / \sigma_{i}=0.73$ and (d) $\sigma / \sigma_{i}>0.62$ 
This value is in fair agreement with the computed value $a_{0}=260 \mathrm{~m} / \mathrm{s}$, considering the elastic deformation of the PVC pipe wall and using Eq. (1). The viscoelastic behavior of the PVC material is not considered in this investigation.

To describe the influence of the cavitating section, a lumped compliance, see Eq. (2), is included in the model to account for the vapor cavity compliance. The compliance is located for a diameter $D$ downstream the obstacle. The simulations are performed with the in-house developed software SIMSEN (http://simsen.epfl.ch/, 2011; Nicolet et al., 2007). A centered finite difference method on a staggered grid with 40 elements is used for the spatial discretization of the pipe, see Fig. 12.

A sensitivity study has been performed and leads us to the conclusion that the selected spatial resolution, $0.026 \mathrm{~m}$, is sufficient for the investigated wave length range $\lambda>0.67 \mathrm{~m}$.

The following set of equations is obtained for $i=1,2, \ldots, 40$ :

$$
\left\{\begin{array}{ll}
\left(1+\frac{a_{0}^{2}}{A_{0}} \frac{K}{\Delta x}\right)^{-1} \frac{d p_{i}}{d t}+\rho_{0} a_{0}^{2} \frac{C_{i+1 / 2}-C_{i-1 / 2}}{\Delta x}=0, & i=30 \\
\frac{d p_{i}}{d t}+\rho_{0} a_{0}^{2} \frac{C_{i+1 / 2}-C_{i-1 / 2}}{\Delta x}=0, & i \neq 30
\end{array}, \frac{d C_{i+1 / 2}}{d t}+\frac{1}{\rho_{0}} \frac{p_{i+1}-p_{i}}{\Delta x}+\frac{f\left|C_{0}\right|}{2 \sqrt{A_{0}}} C_{i+1 / 2}=0 .\right.
$$

The cavity compliance $K$ is added only for the node which corresponds to the vapor cavity position $i=30$. Similar formulation has been successfully used to describe the dynamic behavior of a Francis turbine at full load operation (Nicolet, 2007). The resulting system of the first order differential equations is given by

$$
\frac{d \vec{X}}{d t}+[M] \vec{X}=\vec{V}
$$

In Eq. (11), $\vec{X}$ is the state variable vector $\left(p_{i}\right.$ and $C_{i}$ for $i=1,2, \ldots, 40$ ). The vector $\vec{V}$ contains the pressure boundary conditions, $p_{\text {in }}$ and $p_{\text {out }}$. The matrix $[M]$ contains the fluid inertia, propagation velocity, cavity compliance and wall friction. The eigenfrequencies of the linearized system are determined from the eigenvalues of the matrix [M] (Alligné et al., 2008). Complex eigenvalues correspond to oscillatory modes. Damping and oscillation frequencies are, respectively, given by the real parts and the imaginary parts of the eigenvalues. The shapes of the corresponding eigenmodes are given by the eigenvectors.

The aim of the one-dimensional hydroacoustic model is to compare the eigenfrequencies and the eigenmode shapes of the cavitating pipe with respect to the experimental results. Therefore, the head losses due to the pipe wall friction and the bluff body drag which only influence the damping are not taken into account for this analysis and, then, we impose for the boundary conditions $p_{\text {in }}=p_{\text {out }}$.

The parameters of the one-dimensional hydroacoustic model are summarized in Table 2 .

\subsection{Eigenfrequency}

Based on the hydroacoustic model, the 1st, 2nd and 3rd eigenfrequencies of the system are plotted versus the cavity compliance values in Fig. 13. The cavity compliance is presented in a logarithmic scale. A nonlinear relation between the eigenfrequencies and $K$ is shown. At low cavity compliance, the 1st eigenfrequency is equal to the eigenfrequency of the pipe free of vapor. At high $K$, the first eigenfrequency drops to a value close to zero and the eigenfrequencies of higher orders tend to constant values. Such a situation has been analyzed by Testud et al. (2007), for developed cavitation regime.

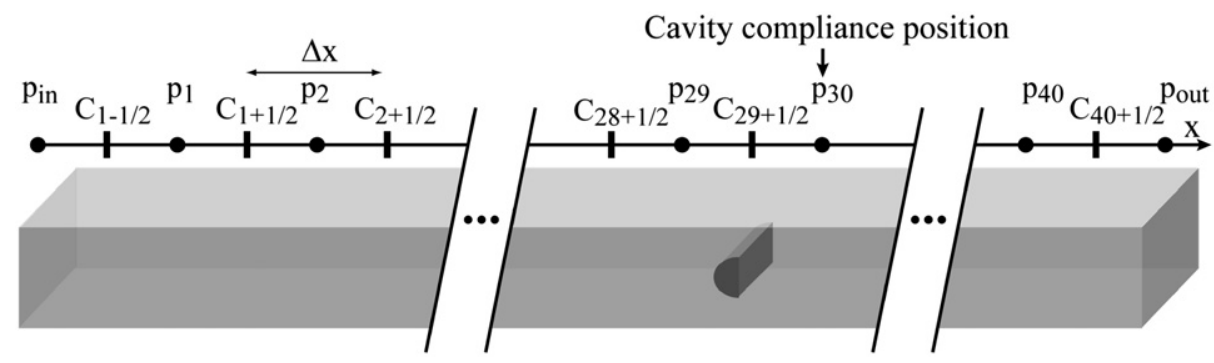

Fig. 12. Pipe space discretization for the SIMSEN simulations.

Table 2

Set-up of the one-dimensional hydroacoustic model.

\begin{tabular}{ll}
\hline Parameter & Value \\
\hline Propagation velocity & $203 \mathrm{~m} / \mathrm{s}$ \\
Friction factor & 0 \\
Inlet BC & $0 \mathrm{~Pa}$ \\
Outlet BC & $0 \mathrm{~Pa}$ \\
Length & $1.05 \mathrm{~m}$ \\
\hline
\end{tabular}




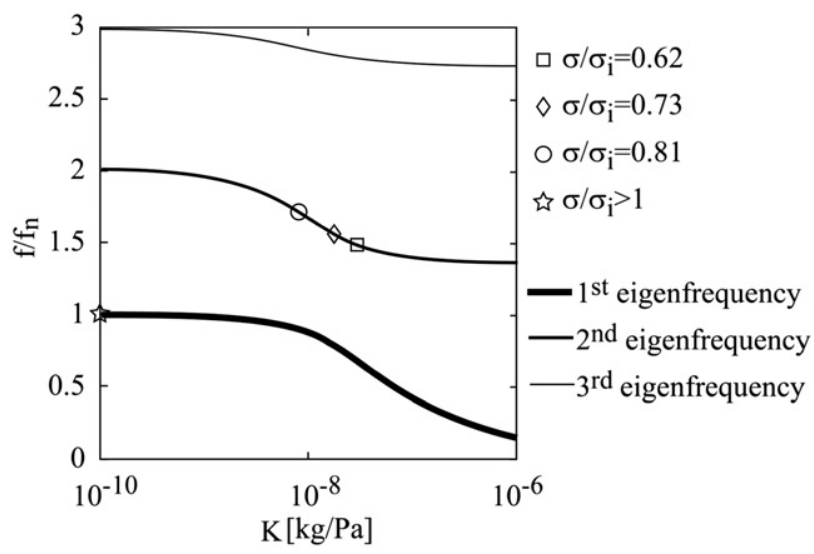

Fig. 13. Influence of the vapor cavity compliance on eigenfrequencies.

Table 3

Relation between the normalized cavitation index and the computed eigenfrequencies.

\begin{tabular}{|c|c|c|c|c|}
\hline$\sigma / \sigma_{i}(-)$ & $\mathrm{K}(\mathrm{kg} / \mathrm{Pa})$ & 1st Eigenfrequency $\left(f / f_{n}\right)$ & 2nd Eigenfrequency $\left(f / f_{n}\right)$ & 3rd Eigenfrequency $\left(f / f_{n}\right)$ \\
\hline$>1$ & 0 & 1 & 2 & 3 \\
\hline 0.81 & $8.24 \times 10^{-9}$ & 0.89 & 1.7 & 2.91 \\
\hline 0.73 & $1.79 \times 10^{-8}$ & 0.75 & 1.55 & 2.85 \\
\hline 0.62 & $3.07 \times 10^{-8}$ & 0.61 & 1.48 & 2.82 \\
\hline
\end{tabular}

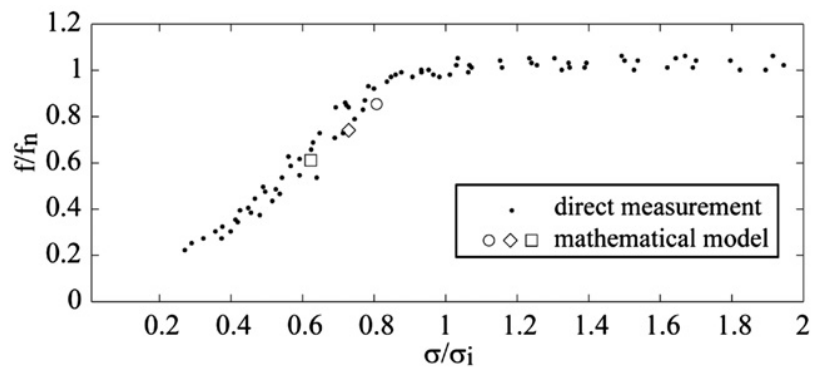

Fig. 14. 1st Eigenfrequency versus cavitation index.

Based on the measured resonant frequencies and the relation between the eigenfrequencies and the cavitation index, the cavity compliance corresponding to the selected cavitation indexes can be evaluated, yielding $K=8.24 \times 10^{-9} \mathrm{~kg} / \mathrm{Pa}$ for $\sigma / \sigma_{i}=0.81 ; K=1.79 \times 10^{-8} \mathrm{~kg} / \mathrm{Pa}$ for $\sigma / \sigma_{i}=0.73$ and $K=3.07 \times 10^{-8} \mathrm{~kg} / \mathrm{Pa}$ for $\sigma / \sigma_{i}=0.62$. The $1 \mathrm{st}$ and 3rd eigenfrequencies can thereafter be estimated from the cavity compliance, see Table 3.

In Fig. 14, the direct measurements of the first eigenfrequency of the system as a function of the cavitation index are compared with the results obtained with the numerical simulations.

The direct measurement of the frequency of the peak due to broadband noise of the source is assumed to match the eigenfrequency of the system for the corresponding cavitation index. The frequency peaks presented in Fig. 10 are plotted versus $\sigma$, solid symbols in Fig. 14.

While for the numerical simulations, the first eigenfrequency is evaluated for the selected cavitation indexes (circle: $\sigma / \sigma_{i}=0.81$; diamond: $\sigma / \sigma_{i}=0.73$; square: $\sigma / \sigma_{i}=0.62$ ). The good agreement leads us to the conclusion that the lumped compliance model is sufficient to describe the influence of the cavitation on the eigenfrequencies of the system.

\subsection{Eigenmodes}

The eigenmodes are directly given by the eigenvectors of the hydroacoustic model. Those modes are compared with the measured pressure fluctuations in Fig. 15 for $\sigma / \sigma_{i}>1, \sigma / \sigma_{i}=0.81, \sigma / \sigma_{i}=0.73$ and $\sigma / \sigma_{i}=0.62$. The frequency response 

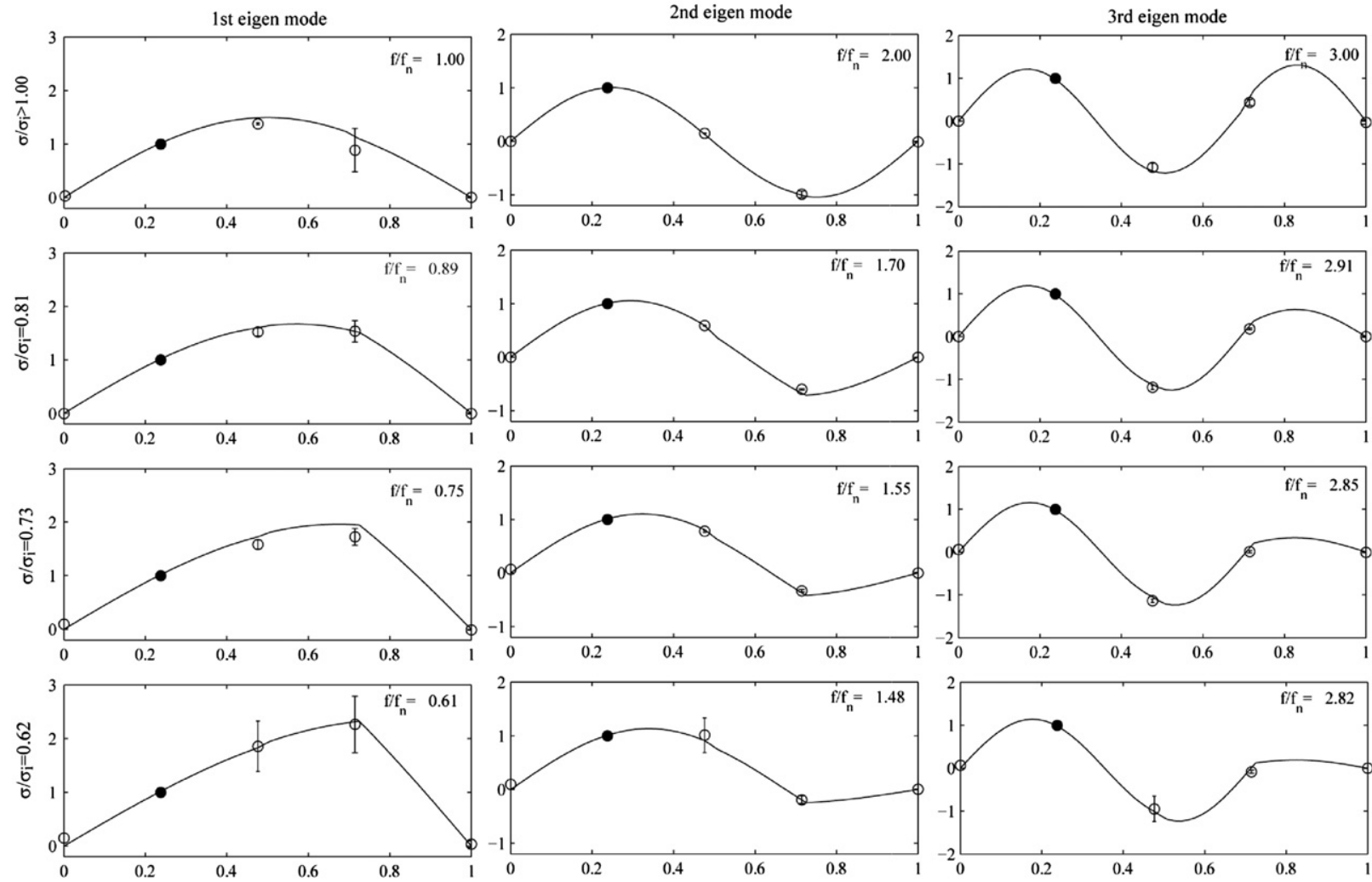

Fig. 15. 1st, 2nd and 3rd pressure eigenmodes for various cavitation indexes. Black point: reference amplitude; error bar: mean value and standard deviation of the transfer functions; lines: one-dimensional hydroacoustic model.

function with the reference sensor $x / L_{\mathrm{TOT}}=0.25$, solid symbol in Fig. 15, is used to determine the distribution of the pressure fluctuations for a given frequency (Testud et al., 2007).

The frequency response function is estimated with the cross power spectral density normalized by the power spectral density at the reference position:

$$
T_{x y}(f)=\frac{P_{y x}(f)}{P_{x x}(f)} .
$$

The frequency response function is computed for each selected cavitation index, the mean values and the standard deviations of the frequency response function are presented for the frequencies corresponding to the first, second and third eigenfrequency values. The different flow velocities at the selected cavitation indexes, see solid symbols in Fig. 4, are used to plot the error bar. To compare the eigenvectors with the measurements, the eigenvectors are normalized with their amplitude at $x / L_{\mathrm{TOT}}=0.25$. The discontinuity produced by the cavity compliance is clearly visible for the eigenvectors at low $\sigma$. The anti-node of the 1st eigenmode is shifted from the middle of the pipe to the vapor cavity position along with the decrease of the cavitation index $\sigma$. For the 2nd eigenmode, the vapor cavity tends to behave as a node of pressure with the increase of vapor compliance. This tendency is amplified for the 3rd eigenmode. At very low $\sigma$, the vapor tends to split the circuit in two parts. The plane waves are fully reflected by the cavity and both sides of the circuit behave as two independent systems. This is valid for all except for the first eigenmode which corresponds to the pulsation of the vapor cavity.

\section{Conclusion}

A wall pressure measurement of hydroacoustic resonance has been performed in a straight PVC pipe in cavitating flow conditions. The flow instability in the wake of a bluff body was successfully exploited as a source of acoustic pressure wave. Its frequency was adjusted with the flow velocity; a linear relation has been measured between the source frequency and the flow velocity, following a constant Strouhal number law. The influence of hydrodynamic cavitation in a limited section of the pipe on the eigenfrequencies of the hydroacoustic system has been investigated in detail. Using broadband noise excitation and resonance, the dependence of the eigenfrequencies on the cavitation index has been demonstrated. The values of the eigenfrequencies decrease in a nonlinear manner with the decrease of the cavitation 
index. A mathematical model based on one-dimensional pipe flow and lumped vapor cavity compliance has been used to analyze this phenomenon. A relation between the cavitation index and the cavity compliance was established. The eigenmodes computed with the mathematical model were in good agreement with the measured distribution of pressure fluctuation at the corresponding eigenfrequencies. Based on the measurement of a single eigenfrequency at a given cavitation index, it was possible to predict the other eigenfrequencies and eigenmodes corresponding to this cavitation index. A lumped compliance included in a hydroacoustic model of the pipe was sufficient to describe the influence of hydrodynamic cavitation on the eigenfrequencies and eigenmodes of the system. This experimental work emphasizes the advantages of one-dimensional hydroacoustic models with lumped compliance for the analysis of pressure fluctuation in circuit with cavitation. The precision of the approach is shown for a hydrodynamic source of acoustic fluctuation featuring cavitation. Such approach is expected to provide valuable results for the interaction between other type of hydrodynamic instabilities such as vortex breakdown and arbitrary hydraulic circuit.

\section{Acknowledgments}

The authors would like to thank particularly ALSTOM Hydro for their financial support and technical assistance. The project is also financially supported by CTI, the Swiss Federal Commission for Technology and Innovation, Grant no. 8330.2 EPRP-IW; CCEM and Swisselectric Research.

\section{References}

Alligné, S., Nicolet, C., Allenbach, P., Kawkabani, B., Simond, J.J., Avellan, F., 2008. Influence of the Francis turbine location under vortex rope excitation on the hydraulic system stability. International Journal of Fluid Machinery and Systems 2 (4), 286-294, October-December 2009, Paper no. O09027S.

Arpe, J., Nicolet, C., Avellan, F., 2009. Experimental evidence of hydroacoustic pressure waves in a Francis turbine elbow draft tube for low discharge conditions. Transactions of ASME, Journal of Fluids Engineering 131, 081102.

Berten, S., Farhat, M., Dupont, P., Avellan, F., 2007. Rotor-stator interaction induced pressure fluctuations: CFD and hydroacoustic simulations in the stationary components of a multistage centrifugal pump. In: Proceedings of the FEDSM2007-37549, San Diego, USA.

Blake, W.K., 1986. Mechanics of Flow-Induced Sound and Vibration. Academic Press, Orlando, USA (Chapter 2).

Bouziad, Y., Farhat, M., Guennoun, F., Kueny, J.L., Avellan, F., Miyagawa, K., 2003. Physical modeling and simulation of leading edge cavitation, application to an industrial inducer, in: Proceedings of the CAV2003, Osaka, Japan.

Bouziad, Y., 2005. Physical Modeling of Leading Edge Cavitation : Computational Methodologies and Application to Hydraulic Machinery, Doctoral Thesis No. 3353, EPFL, Lausanne, Switzerland.

Brennen, C.E., Acosta, A.J., 1976. The dynamic transfer function for a cavitating inducer. Transactions of ASME, Journal of Fluids Engineering 98, 182-191. Curle, N., 1955. The influence of solid boundaries upon aerodynamic sound. Proceedings of the Royal Society of London, Series A $231,505-514$.

Eid, M., Ziada, S., 2011. Vortex shedding and acoustic resonance of single and tandem finned cylinders. Journal of Fluids and Structures 27 (7), $1035-1048$

Farhat, M., Natal, S., Avellan, F., Paquet, F., Lowys, P.Y., Couston, M., 2002. Onboard measurements of pressure and strain fluctuations in a model of low head Francis turbine, Part 1: instrumentation. In: Proceedings of the 21st AIRH Symposium on Hydraulic Machinery and Systems, Lausanne, Switzerland, vol. 2, pp. 903-910.

Ghidaoui, M.S., Zhao, M., McInnis, D.A., Axworthly, D.H., 2005. A review of water Hammer theory and practice. Applied Mechanics Reviews 58, 49-76.

Hachem, F., Schleiss, A., 2011. A review of wave celerity in frictionless and axisymmetrical steel-lined pressure tunnels. Journal of Fluids and Structures 27 (2), 311-328.

Hassis, H., 1999. Noise caused by cavitating butterfly and monovar valves. Journal of Sound and Vibration 225 (3), $515-526$.

Hirschberg, A., Rienstra, S., 2004. An Introduction to Aeroacoustics. Technical Report, Eindhoven University of Technology, Eindhoven, Netherlands.

Inoue, O., Hatakeyama, N., 2002. Sound generation by a two-dimensional circular cylinder in a uniform flow. Journal of Fluid Mechanics 471 , 285-314.

Nicolet, C., 2007. Hydroacoustic Modeling and Numerical Simulation of Unsteady Operation of Hydroelectric Systems, Doctoral Thesis No. 3751, EPFL, Lausanne, Switzerland.

Nicolet, C., Greiveldinger, B., Hérou, J.-J., Kawkabani, B., Allenbach, P., Simond, J.-J., Avellan, F., 2007. High-order modeling of hydraulic power plant in islanded power network. IEEE Transactions on Power Systems 22 (4), 1870-1880.

Ota, T., Okamoto, Y., Yoshikawa, H., 1994. A correction formula for wall effects on unsteady forces of two-dimensional bluff bodies. Transactions of ASME, Journal of Fluids Engineering 116, 414-418.

Reethof, G., 1978. Turbulence-generated noise in pipe flow. Annual Review of Fluid Mechanics 10, $333-367$.

Rubin, S., 1966. Longitudinal instability of liquid rockets due propulsion feedback (POGO). Journal of Spacecraft and Rockets 3, $1188-1195$.

Testud, P., Moussou, P., Hirschberg, A., Aurégan, Y., 2007. Noise generated by cavitating single-hole and multi-hole orifices in a water pipe. Journal of Fluids and Structures 23, 163-189.

Tijsseling, A.S., 1996. Fluid-structure interaction in liquid-filled pipe systems: a review. Journal of Fluids and Structures 10, 109-146.

Watanabe, S., Brennen, C.E., 2003. Dynamics of a cavitating propeller in a water tunnel. Transactions of ASME, Journal of Fluids Engineering 125, 283-292.

Welch, P., 1967. The use of fast Fourier transform for the estimation of power spectra: a method based on time averaging over short, modified periodograms. IEEE Transactions on Audio and Electroacoustics 15, 70-73.

Williams, J.E., 1969. Hydrodynamic noise. Annual Review of Fluid Mechanics 1, 197-222.

Williamson, C.H.K., 1996. Vortex dynamics in the cylinder wake. Annual Review of Fluid Mechanics 28, 477-539.

Wylie, E.B., Streeter, V.L., 1993. Fluid Transients in Systems. Prentice Hall, Englewood Cliffs.

Young, J.O., Holl, J.W., 1966. Effects of cavitation on periodic wakes behind symmetric wedges. Transactions of ASME, Journal of Basic Engineering $88,163-176$ 